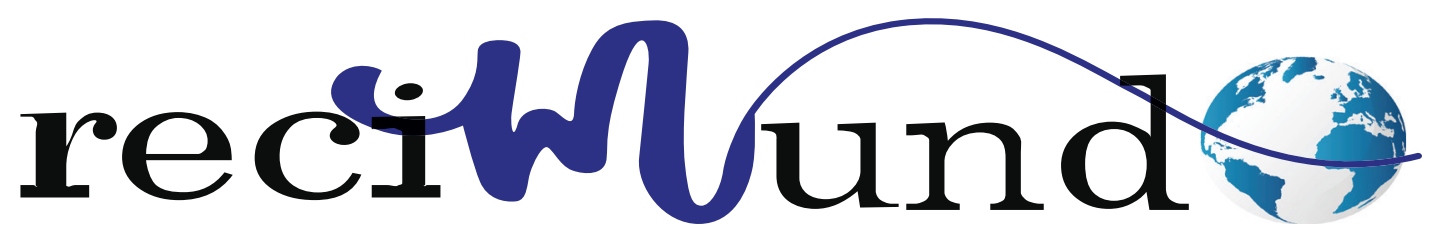

Revista Científica Mundo de la Investigación y el Conocimiento

DOI: 10.26820/recimundo/4.(4).noviembre.2020.138-147

URL: http://recimundo.com/index.php/es/article/view/933

EDITORIAL: Saberes del Conocimiento

REVISTA: RECIMUNDO

ISSN: 2588-073X

TIPO DE INVESTIGACióN: Artículo de revisión

CóDIGO UNESCO: 32 Ciencias Médicas

PAGINAS: $138-147$

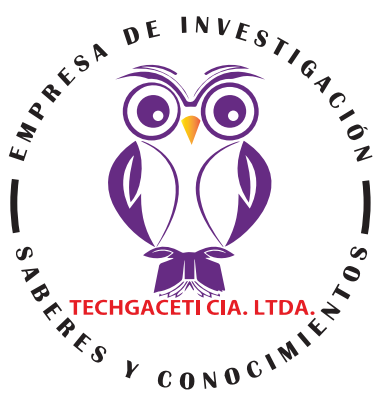

\title{
Trastornos fonoaudiológicos en pacientes hospitalizados
}

Speech and language disorders in hospitalized patients

Distúrbios de fala e linguagem em pacientes hospitalizados

José Estefan Delgado Vélez'; Steven Sebastián Flores Posligua²

RECIBIDO: 08/08/2020 ACEPTADO: 02/09/2020 PUBLICADO: 10/11/2020

1. Postgradista en Seguridad y Salud Ocupacional; Licenciado en Terapia de Lenguaje; Experiencia en Ministerio de Salud y Ministerio de Inclusión Económica; Presidente de Asociación de Fonoaudiólogos; Calceta, Ecuador; stefandelgado. m29a@gmail.com; iD https://orcid.org/0000-0001-8740-0207

2. Fonoaudiólogo; Experiencia en Salud tanto en Centros Hospitalarios y Atención Ambulatoria a Personas con Discapacidad; Jaramijó, Ecuador; steven.flores1995@gmail.com; iD https://orcid.org/0000-0002-0394-6526

\section{CORRESPONDENCIA}

José Estefan Delgado Vélez

stefandelgado.m29a@gmail.com

Calceta-Ecuador 


\section{RESUMEN}

Los trastornos fonoaudiológicos comprenden alteraciones de voz, lenguaje, audición, habla y deglución, éstas condiciones pueden presentarse de forma aislada o con mayor frecuencia, secundario a una condición orgánica o neurológica que comprometa una o más de las áreas mencionadas. La estadía del paciente hospitalizado en las diferentes áreas de servicio depende de la patología base y de los eventos posteriores a éste, que en mayoría de ocasiones es el principal determinante del tiempo de permanencia del individuo en la sala de hospitalización. El objetivo de la investigación es dar a conocer la incidencia de trastornos fonoaudiológicos en pacientes hospitalizados en el área de medicina interna, la presente investigación se lleva a cabo en un nosocomio de segundo nivel de atención, con una duración de 7 meses, el número total de atendidos en esta área durante el tiempo de estudio fue de 212, mientras que 158 fueron pacientes que presentaron un trastorno fonoaudiológico. Se obtuvieron datos provenientes de las historias clínicas de cada uno de los pacientes y se realizaron valoraciones por el área de fonoaudiología. El trastorno deglutorio fue el que tuvo la prevalencia más alta entre la muestra estudiada, con un total de 120 casos causados en 38 veces por neumonía, mientras que el trastorno del habla se presentó en menor cantidad.

Palabras clave: Fonoaudiología, trastornos de deglución, neumonía.

\section{ABSTRACT}

Speech and language disorders include voice, language, hearing, speech and swallowing disorders. These conditions can occur in isolation or more frequently, secondary to an organic or neurological condition that compromises one or more of the aforementioned areas. The stay of the hospitalized patient in the different service areas depends on the underlying pathology and the events after it, which in most cases is the main determinant of the individual's time spent in the hospitalization room. The objective of the research is to publicize the incidence of speech therapy disorders in hospitalized patients in the area of internal medicine, the present investigation is carried out in a second-level care hospital, with a duration of 7 months, the total number Of attended in this area during the study time was 212, while 158 were patients who presented a speech-language disorder. Data were obtained from the medical records of each of the patients and assessments were made by the speech therapy area. Swallowing disorder was the one with the highest prevalence among the sample studied, with a total of 120 cases caused 38 times by pneumonia, while speech disorder occurred in lesser quantity.

Keywords: Speech, Language and Hearing Sciences, Deglutition Disorders, Pneumonia.

\section{RESUMO}

Os distúrbios da fala e da linguagem incluem distúrbios de voz, linguagem, audição, fala e deglutição. Essas condições podem ocorrer de forma isolada ou, mais freqüentemente, secundárias a uma condição orgânica ou neurológica que comprometa uma ou mais das áreas citadas. A permanência do paciente internado nas diferentes áreas de serviço depende da patologia de base e dos eventos subsequentes, que na maioria das vezes é o principal determinante do tempo de permanência do indivíduo na sala de internação. O objetivo da pesquisa é divulgar a incidência de distúrbios fonoaudiológicos em pacientes internados na área de clínica médica, a presente investigação é realizada em um hospital de segundo nível de atendimento, com duração de 7 meses, número total De atendidos nessa área durante o tempo de estudo foi de 212, enquanto 158 eram pacientes que apresentavam algum distúrbio fonoaudiológico. Os dados foram obtidos nos prontuários de cada um dos pacientes e as avaliações foram feitas pela área de Fonoaudiologia. O distúrbio da deglutição foi o que apresentou maior prevalência na amostra estudada, com um total de 120 casos causados 38 vezes por pneumonia, enquanto o distúrbio da fala ocorreu em menor quantidade.

Palavras-chave: Fonoaudiologia, Distúrbios da Deglutição, Pneumonia. 


\section{Introducción}

El reconocimiento del fonoaudiólogo en áreas de hospitalización como un miembro clave en la pronta rehabilitación en este tipo de pacientes y áreas de hospitalización es fundamental para lograr una mejor intervención multidisciplinaria, elaborando planes de intervención temprana con el objetivo de prevenir y reducir complicaciones que a futuro pueden desencadenar problemas más severos, evitando el menor tiempo posible una estadía hospitalaria que se caracteriza a procesos clínicos, alteraciones en patrones miofuncionales orofaciales, alimentación, función deglutoria y en el caso de lactantes reflejos primarios (1)

La intervención fonoaudiológica en los hospitales es relativamente reciente debido al poco conocimiento del accionar y desempeño que realiza este profesional en dichas áreas, especialmente en lo que respecta a los pacientes hospitalizados (2), por lo que se tiene desconocimiento del accionar del fonoaudiólogo en dichas áreas.

A la par ha crecido la preocupación por brindar servicios de calidad que integren un equipo multidisciplinar especializado en la detección, evaluación diagnostica y tratamiento de esta (3), consiguiendo así una recuperación más acelerada optimizando las funciones deterioradas, logrando que sea el paciente/usuario y no el desorden o algún tipo de complicación a largo plazo el motivo de reingreso hospitalario. Una adecuada y temprana intervención multidisciplinaria otorga mejores cualidades de recuperación post-hospitalización.

Éste estudio tiene como objetivo conocer la prevalencia de los trastornos fonoaudiológicos en pacientes hospitalizados en el servicio de medicina interna, determinando las características sociodemográficas, patologías desencadenantes de las alteraciones y los trastornos específicos encontrados en los individuos investigados.

\section{Material y método}

El tipo de población que se utilizó para la presente investigación es de corte trasversal y retrospectivo, lo que nos permite estimar la magnitud y distribución del problema. La misma que se realizó en un centro de salud de segundo nivel de atención, en el servicio de medicina interna, el cual tuvo un tiempo de duración de 7 meses. Se eligió una población de tipo finita, conformada por los pacientes ingresados en el área de medicina interna con un total de 212 pacientes. De esta población que fue tomada para el estudio, se emplearon valoraciones fonoaudiológicas dando como resultado una muestra patológica de 158 individuos, con características sociodemográficas a detallar posteriormente.

Los objetos de estudio están representados en noventa (90) hombres y sesenta y ocho (68) mujeres que representa a la cantidad de individuos que previo a las valoraciones dieron positivos a trastornos fonoaudiológicos, dando un total de 158 pacientes en edades que oscilan desde los 20 a 65 años aproximadamente. Los criterios de muestreo consistieron en segregar los pacientes que no presentaban ningún déficit o alteración a nivel de cognición, habla, audición, lenguaje, deglución o voz.

Para la recolección de datos se emplearon entrevistas directas dirigidas a cuidadores, pacientes y profesionales que laboran en el servicio de medicina interna, se recaudó información de las historias clínicas y su respectiva valoración fonoaudiológica.

Al amplio abanico patológico que presentaba el área de medicina interna, permitió observar diferentes tipos de condiciones, que fueron desde enfermedades respiratorias como la tuberculosis, hasta padecimientos que comprometen el sistema nervioso, como por ejemplo los traumatismos craneoencefálicos y los eventos cerebrovasculares, en las que se presentan secuelas de tipo motor y sensitivo. 


\section{Resultados}

La población presente en el área de medicina interna fue de 212, una vez valorados por el servicio de fonoaudiología se obtuvieron 158 pacientes con trastornos específicos, del habla, lenguaje, deglución o voz.

\begin{tabular}{|l|l|l|}
\hline SEXO & F & $\%$ \\
\hline Masculino & 102 & 65 \\
\hline Femenino & 56 & 35 \\
\hline TOTAL & 158 & 100 \\
\hline
\end{tabular}

Tabla 1. Categorización sociodemográfica de la población por sexo.

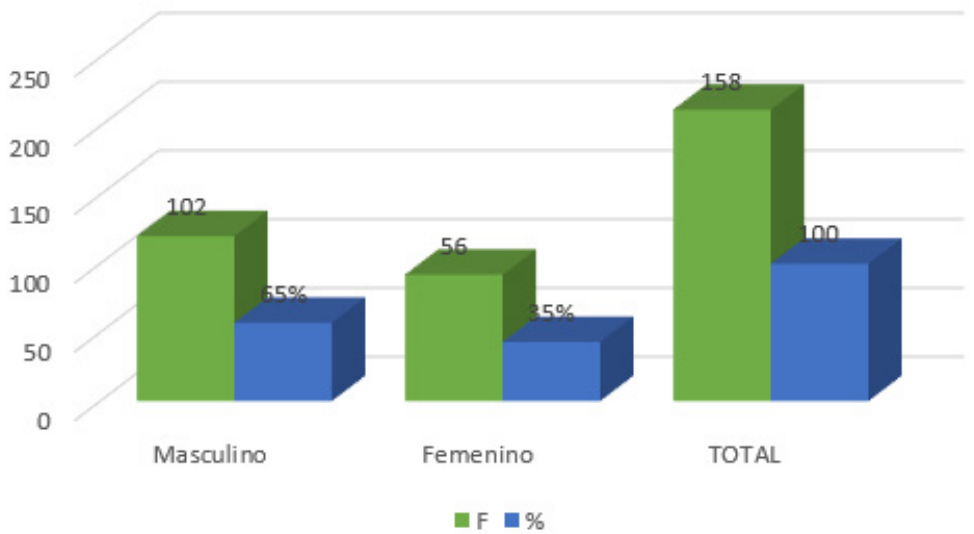

Gráfico 1. Categorización sociodemográfica de la población por sexo.

La prevalencia del sexo masculino con un porcentaje de $65 \%$ sobre el sexo femenino que consta del $35 \%$ de pacientes hospita- lizados en el servicio de medicina interna, determinando mayor número en el sexo masculino.

\begin{tabular}{|l|l|l|}
\hline \multicolumn{3}{|c|}{ EDADES } \\
\hline AÑOS & FRECUENCIA & $\%$ \\
\hline $20-39$ & 31 & 20 \\
\hline $40-59$ & 88 & 55 \\
\hline $60-+$ & 39 & 25 \\
\hline TOTAL & $\mathbf{1 5 8}$ & $\mathbf{1 0 0}$ \\
\hline
\end{tabular}

Tabla 2. Categorización sociodemográfica de la población por edades. 


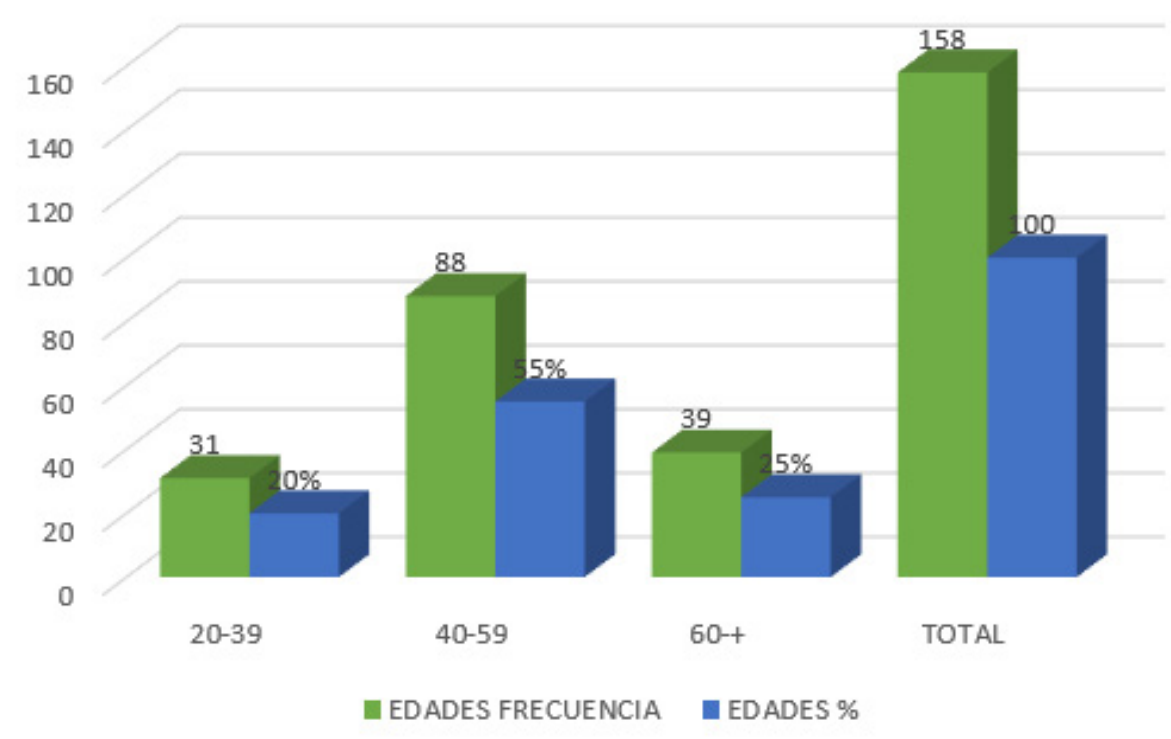

Gráfico 2. Categorización sociodemográfica de la población por edades.

Los datos afirman que la población más afectada comprende un rango de 40 a 59 años que representa un 55\%, en cambio el porcentaje de menor cantidad que padece un trastorno fonoaudiológico oscila entre los 20-39 años con un porcentaje del 20\%. Garrido (1995) afirma que la edad media se sitúa entre los 55 años y se corresponde con patología afásica, encontrándose, en el caso de las afasias mixtas, una edad media de aparición más tardía para las mujeres (los 69 años para la mujer y los 55 años para el hombre).

\begin{tabular}{|l|c|c|c|}
\hline \multirow{2}{*}{ Patologías } & \multicolumn{2}{|c|}{ Sexo } & \multirow{2}{*}{ Total pacientes } \\
\cline { 2 - 3 } & $\mathbf{M}$ & $\mathbf{F}$ & \\
\hline Traumatismo craneoencefálico & 20 & 11 & 31 \\
\hline Accidente cerebrovascular & 21 & 9 & 30 \\
\hline Paciente traqueostomizado & 23 & 14 & 37 \\
\hline Neumonías & 25 & 13 & 38 \\
\hline Tuberculosis & 13 & 9 & 22 \\
\hline Total & $\mathbf{1 0 2}$ & $\mathbf{5 6}$ & $\mathbf{1 5 8}$ \\
\hline
\end{tabular}

Tabla 3. Patología base de trastorno fonoaudiológico por sexo. 


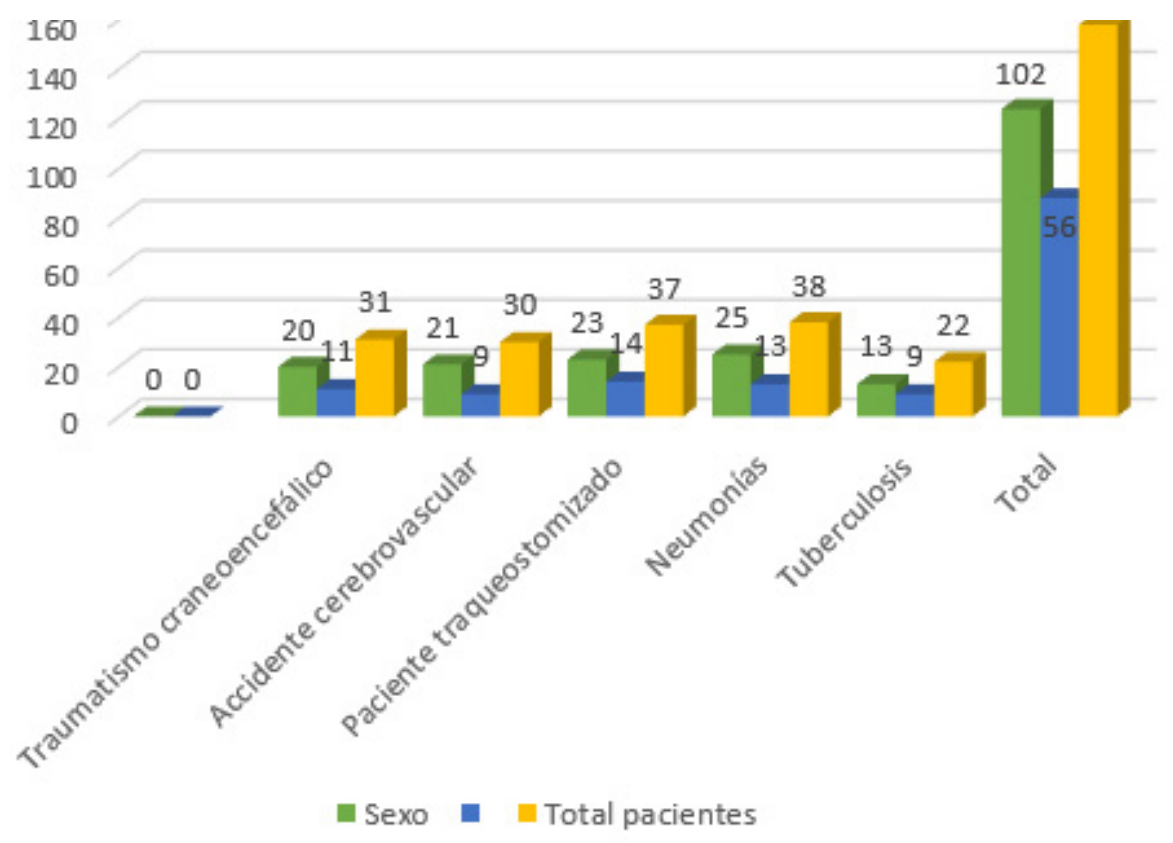

Gráfico 3. Patología base de trastorno fonoaudiológico por sexo

La traqueotomía se convirtió en la causa más frecuente para que el paciente pueda desarrollar una patología fonoaudiológica, le siguen eventualidades que comprometen al sistema vascular del cerebro tales como los traumatismos y los accidentes cerebrovasculares y en menor cantidades, patologías respiratorias como la tuberculosis

\begin{tabular}{|l|c|c|}
\hline & $\mathbf{F}$ & $\%$ \\
\hline Deglución & $\mathbf{1 2 0}$ & $\mathbf{7 6}$ \\
\hline Voz & $\mathbf{2 1}$ & $\mathbf{1 3}$ \\
\hline Habla & $\mathbf{7}$ & $\mathbf{4}$ \\
\hline Lenguaje & $\mathbf{1 0}$ & $\mathbf{7}$ \\
\hline Total & $\mathbf{1 5 8}$ & $\mathbf{1 0 0}$ \\
\hline
\end{tabular}

Tabla 4. Trastornos fonoaudiológicos presentes en la muestra.

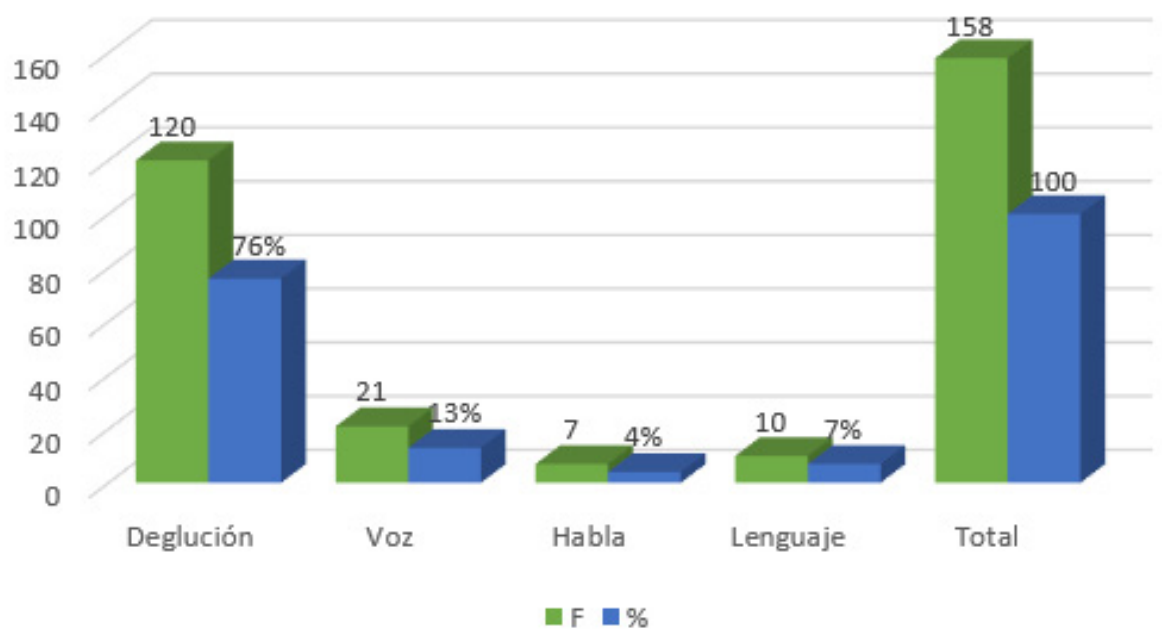

Gráfico 4. Trastornos fonoaudiológicos presentes en la muestra. 
El trastorno deglutorio es la condición de mayor incidencia en el servicio de medicina interna con 120 casos equivalentes a un $76 \%$ del total estudiado, siguiendo con los trastornos de voz con un 13\%, lenguaje con un $7 \%$ y habla con la menor cantidad de entre todos los trastornos con el $4 \%$.

\begin{tabular}{|l|l|l|l|l|l|l|l|l|l|}
\hline \multirow{2}{*}{ Patologías } & \multicolumn{7}{|c|}{ Trastornos fonoaudiológicos } & \multirow{2}{*}{$\begin{array}{c}\text { Total } \\
\text { pacientes }\end{array}$} \\
\cline { 2 - 11 } & Deglución & $\%$ & Voz & $\%$ & Habla & $\%$ & Lenguaje & $\%$ & \\
\hline TCE & 17 & 14 & 4 & 19 & 4 & 57 & 6 & 60 & 31 \\
\hline ACV & 20 & 17 & 3 & 14 & 3 & 43 & 4 & 40 & 30 \\
\hline Traqueostomía & 23 & 19 & 14 & 67 & 0 & 0 & 0 & 0 & 37 \\
\hline Neumonía & 38 & 32 & 0 & 0 & 0 & 0 & 0 & 0 & 38 \\
\hline TB & 22 & 18 & 0 & 0 & 0 & 0 & 0 & 0 & 22 \\
\hline Total & 120 & 100 & 21 & 100 & 7 & 100 & 10 & 100 & 158 \\
\hline
\end{tabular}

Tabla 5. Relación entre trastornos fonoaudiológicos y patologías base.

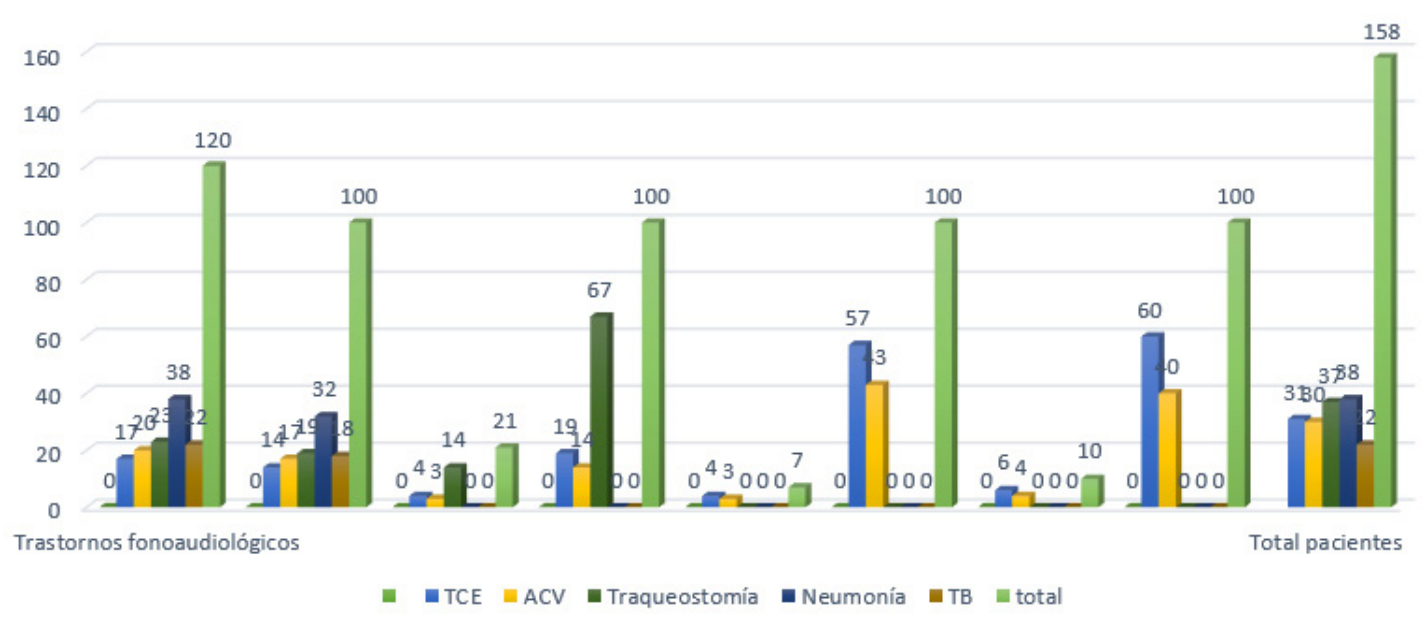

Tabla 6. Relación entre trastornos fonoaudiológicos y patologías base

Se observa que dichos trastornos tienen diferentes agentes causales, ya sean patologías a nivel del tracto respiratorio o daños a

\section{Discusión}

Una vez expuestas las variables, el estudio determina que de entre los pacientes hospitalizados en el servicio de medicina interna, destacamos al profesional de fonoaudiología, especialidad dentro del ámbito de la medicina como encargado del diagnóstico, tratamiento y rehabilitación de alteraciones que afecten a las habilidades comunicativas nivel cerebral, los cuales son los principales causantes. Podemos definir al trastorno deglutorio como el de mayor incidencia.

como lenguaje, cognición, habla, audición y funciones como respiración, alteraciones de la voz y deglución en pacientes de todas las edades (4). En países como Colombia defienden esta profesión y la plantean de carácter científico, cuyos servicios pueden prestarse donde el conocimiento y aporte disciplinario profesional de la fonoaudiología sea requerido (5), siendo de conveniencia para beneficio social, con el fin de bus- 
car alternativas y propuestas en la mejoría pronta del paciente.

En la investigación los individuos de sexo masculino se presentaron en mayor número que el femenino, el rango que oscila entre los 40 a 59 años de edad, fue el más afectado por las patologías causantes de la hospitalización, de acuerdo con estudios realizados, existe una tasa de incidencia de 25,33 en individuos de 55 a 64 años de edad que han padecido una eventualidad cerebrovascular de etiología cardioembólica (6). El rango de edad para padecer de una eventualidad vascular cerebral comprende los 50 años en adelante, ya que entre mayor edad y con factores de riesgos previos, es mayor la posibilidad de presentarse esta condición. Conocer los factores de riesgos, la atención multidisciplinaria a tiempo y sobre todo la planificación y ejecución de un eficaz plan de tratamiento puede reducir las secuelas de estas condiciones (7).

Las enfermedades que tienen como consecuencia daño cerebral son desencadenantes de ocasionar alteraciones, sintomatología, etiología, y cronicidad, poniendo en peligro la vida del paciente, sumándole a esto el tiempo prolongado de estadía hospitalaria son factores principales de trastornos fonoaudiológicos lo cual evita una pronta recuperación, aumentando el riesgo de complicación, siendo los eventos cerebrovasculares una de las primeras causas de discapacidad y ocupa el segundo lugar de causa de muerte a nivel mundial (8). En las últimas décadas se ha observado un aumento en la frecuencia de realización de la traqueotomía en pacientes ventilados, lo cual es uno de los desencadenantes de afectar funciones como lo son la deglución e inclusive cualidades de la voz, trastornos fonoaudiológicos muy comunes en zonas de hospitalización, a nivel deglutorio altera la mecánica de la deglución y del reflejo tusígeno (9).

Los resultados obtenidos durante esta investigación que arrojaron las entrevistas de- muestran que en áreas de hospitalización los trastornos fonoaudiológicos tienen una gran demanda, mayor aun sin el profesional encargado de corregir y proporcionar una rehabilitación integral, basándose en una correcta evaluación, diagnostico, rehabilitación, promoción de la salud y prevención de los trastornos del lenguaje, habla, deglución, audición, vOz, y comunicación con el fin de mejorar habilidades y funciones comunicativas y alimentarias, incluso se rescata de las actas del XXV Congreso de Logopedia, Foniatría y audiología (10). Cabe tambien recalcar que el personal médico que labora en la institución notó y coincidió la importancia de incluir a un fonoaudiólogo en estas áreas, considerando a la prevención como punto inicial para evitar complicaciones o patologías a futuro. La intervención fonoaudiológica temprana en pacientes hospitalizados ha sido poco considerada en el equipo multidisciplinario, restándole importancia y en el peor de los casos habiendo decrecimiento en la calidad de vida del paciente. Siendo las traqueotomías uno de los procedimientos quirúrgicos más comunes en estas áreas, funciones como la deglución se ven seriamente comprometidas, afectando componentes sensoriomotores, lo que compromete la fase oral y faríngea, presentando cuadros de disfunciones orofaríngeas como regurgitación, incapacidad para expulsar secreciones, carraspeo y disfonía corriendo con el riesgo de padecer broncoaspiraciones, las cuales si no se corrigen, posteriormente conllevarían a infecciones respiratorias producto de neumonías aspirativas y en el peor de los casos la muerte prematura del paciente. El tubo orotraqueal mantiene abierta la glotis durante un tiempo prolongado, alterando el normal funcionamiento y relación respiración-deglución al volverlos independiente, normalmente estos deberían ser completamente dependientes, de esta manera los reflejos de protección de la vía aérea, así como disminución de la respuesta motora y acortamiento de tiempos laríngeos, limitación de movimientos en laringe y la musculatura faríngea, causando atrofia 
muscular, debilidad, rigidez de la lengua, faringe y laringe. Se pudo evidenciar alteraciones de voz, que en la realización de procedimientos ajenos a las patologías bases alteran la función vocal de manera anatómica y fisiológica, habiendo otros desordenes como la discontinuación de patrones respiratorios y optando por establecer modelos incorrectos, el volumen inspiratorio decrece afectando la musculatura inspirativa y espirativa.

La implementación de estrategias fonoaudiológicas tempranas tiene como objetivo identificar mejoras para tratar el tipo de trastorno que desarrolle el paciente, ajustando un plan de intervención individualizado, independiente de la etiología y gravedad del trastorno que padezca aplicando una estrategia terapéutica adecuada, evitando así un mayor tiempo prolongado en hospitalización, optimizando la calidad de vida del paciente (11).

\section{Bibliografía}

1. Ayala Patricia MGY. Realidad laboral del fonoaudiólogo en la unidad de cuidados intensivos. Signos Fónicos. 2015 Junio; I(1).

2. Rodriguez-Padovani Aline PMDDMLFdAC. Protocolo de terapia del habla para la evaluación del riesgo de disfagia (PARD). Revista de la sociedad Brasileña de logopedia. 2007 Julio; XII(3).

3. Leonard Rebecca KK. Dysphagia Assessment and Treatment Planning: A Team Approach. Cuarta ed. San Diego: Plural Publishing; 2018.

4. Ramirez R SACMS. Papel del fonoaudiólogo en el área de salud mental: Una experiencia profesional en el hospital militar central. Revista Med. 2009 Enero; XVII(1).

5. Allignani Gabriela GGMI. El rol del fonoaudiólogo en un hospital materno infantil. Revista del hospital materno infantil Ramón Sardá. 2010; XXVIIII(1).

6. González Francisca LPOV. Incidencia poblacional, características epidemiológicas y desenlace funcional de pacientes con ataque cerebrovascular isquémico y afasia. Revista Médica Chile. 2017.

7. Gaudiano Javier GDGMCVCAPRRVSMVL. Epidemiológica del ataque cerebro vascular en un hospital universitario. Revista Uruguaya de medicina interna. 2019 Mayo; II(24-31).

8. Murray CJ LA. Mortality by cause for eight regions of the world: Global Burden of Disease Study. The Lancet. 1997 Mayo; III(349).

9. A.G. Badillo Melgar MRJGEVGAGH. Manejo del paciente traqueostomizado, cánulas y aplicación de fármacos inhalados. In José. SC. Manual de diagnóstico y terapéutica en neumología. Tercera ed. Sevilla: Neumosur.

10. Martinez Luis CCLMHRMLCMMC. XXV Congreso de logopedia, foniatría y audiología. In La logopedia en Iberoamérica; 2006; Granada.

11. Delgado E. EVENTO CEREBROVASCULAR ISQUÉMICO ATEROTROMBÓTICO: ESTUDIO DE CASO. uct [Internet]. 10 de agosto de 2020 [citado 1 de septiembre de 2020];24(103):28-4. Disponible en: https://www.uctunexpo.autanabooks.com/ index.php/uct/article/view354 


\section{CITAR ESTE ARTICULO:}

Delgado Vélez, J. E., \& Flores Posligua, S. S. (2020). Trastornos fonoaudiológicos en pacientes hospitalizados. RECIMUNDO, 4(4), 138-147. https://doi.org/10.26820/recimundo/4.(4).noviembre.2020.138-147 